\title{
Statistical and simulation study on the separation in Junction Frequencies between ordinary $(0)$ and extraordinary $(X)$ wave in oblique ionograms
}

FengJuan Sun

Wuhan University https://orcid.org/0000-0002-6838-398X

XianRong Wan ( $D$ xrwan@whu.edu.cn )

https://orcid.org/0000-0002-1619-7835

HongBo Zhang

CRIRP: China Research Institute of Radio Wave Propagation

Bao Zhou

CRIRP: China Research Institute of Radio Wave Propagation

\section{PanPan Ban}

CRIRP: China Research Institute of Radio Wave Propagation

HongYan Cao

CRIRP: China Research Institute of Radio Wave Propagation

Full paper

Keywords: Ionosphere, Oblique sounding, Oblique ionogram, Junction frequency, Ordinary wave,

Extraordinary wave

Posted Date: February 9th, 2022

DOI: https://doi.org/10.21203/rs.3.rs-1322307/v1

License: (c) (i) This work is licensed under a Creative Commons Attribution 4.0 International License.

Read Full License 


\section{Title page:}

2 Title: Statistical and simulation study on the separation in Junction Frequencies

3 between ordinary $(O)$ and extraordinary $(X)$ wave in oblique ionograms

4 Author \#1: Sun FengJuan, Radio Detection Research Center, School of Electronic and

5 Information, Wuhan University, Wuhan 430072, China; China Research Institute of

6 Radio-wave Propagation, 36 Xianshan Road, Chengyang District 266107 QingDao,

$7 \quad$ China, zb22501@163.com

8 Author \#2: Wan XianRong, Radio Detection Research Center, School of Electronic and

9 Information, Wuhan University, Wuhan 430072, China, xrwan@whu.edu.cn

10 Author \#3: Zhang HongBo, China Research Institute of Radio-wave Propagation, 36

11 Xianshan Road, Chengyang District 266107 QingDao, China, ssrs_nklee@163.com

12 Author \#4: Zhou Bao, China Research Institute of Radio-wave Propagation, 36

13 Xianshan Road, Chengyang District 266107 QingDao, China, roky510@sohu.com

14 Author \#5: Ban PanPan, Xidian University, Xian 710068, China; China Research

15 Institute of Radio-wave Propagation, 36 Xianshan Road, Chengyang District 266107

16 QingDao, China, bpp432@163.com

17 Author \#6: Cao Hongyan, China Research Institute of Radio-wave Propagation, 36 
18 Xianshan Road, Chengyang District 266107 QingDao, China, xxchy22@126.com

19 This is the corresponding author: Wan XianRong, xrwan@whu.edu.cn 


\section{Abstract}

22 The most important aim in interpreting an oblique ionogram is to obtain the accurate

23 Junction Frequencies (JFs) of the ordinary $(\mathrm{O})$ and extraordinary $(\mathrm{X})$ mode. This

24 requires the correct identification of $\mathrm{O}$ - and $\mathrm{X}$-mode traces, so it is very helpful and

25 worthy to grasp the relative position between the two modes. In this paper, a statistical

26 and simulation study of the separation in JFs between O- and X-wave is carried out

27 based on observed oblique ionograms over three mid-latitude paths within China and a

28 3-D ray tracing program. The dependences on local time, season, geomagnetic activity,

29 solar activity, direction and length of propagation, $\mathrm{O}$-wave JF and group path are

30 investigated. The main conclusions are as follows : (a) the separation on east-west path

31 is susceptible on ionospheric variability, while the separation on north-south path does

32 not show a significant correlation with local time and season;(b) a general diurnal

33 tendency and a summer anomaly on east-west propagation are first proposed and

34 discussed, the latter may be related to the effect of the lower layers; (c)the separation

35 varies approximately as the cosine function of the propagation direction owning two

36 maximums at north-south direction and two minimums at east-west direction in 
37 mid-latitude region of China; (d) the variation patterns of the separation with the

38 propagation length are obviously not the same in different directions. For the east-west

39 propagation path, the separation decreases to a minimum near ground range of $2000 \mathrm{~km}$

40 and then increases very slowly with increasing ground range, while it monotonically

41 increases for north-south propagation path.

\section{Keywords}

43 Ionosphere; Oblique sounding; Oblique ionogram; Junction frequency; Ordinary wave;

44 Extraordinary wave

45 Main Text

461 Introduction

47 Oblique incidence ionospheric sounding(Davies 1990), which transmitter and receiver

48 locate several hundreds or thousands of kilometers apart, is a powerful tool for

49 monitoring the prevailing propagation conditions in real time and is also important for

50 tracking ionospheric effects caused by space weather events. It offers several important

51 advantages over vertical sounding, such as the opportunity of monitoring the ionosphere 
across large, otherwise inaccessible areas such as ocean surfaces and the ability of a

53 receiver to detect signals from many different directions (Ippolito et al. 2015). So it is

54 widely used for propagation predictions of high frequency (HF) radio communications,

55 over-the-horizon radar localization and for basic research purposes (Rao 1973; Yau et al.

56 2006;Chemogor et al. 2020;Rogov et al. 2021). Heitmann et al.(2018) regarded oblique

57 sounding was the most efficient tool for the operational determination of the HF channel

58 characteristics.

59 The oblique ionogram, a group path or time delay versus operating frequency record,

60 contains useful information regarding the state of the ionosphere over the range of the

61 signals traverse (Reily and Kolesar 1989; Phanivong et al. 1995; Ippolito et al. 2015;).

62 The important characteristics like the LOF (the Lowest observed frequency), MOF (the

63 Maximum observed frequency), FMUF (the highest useable frequency of one-hop F

64 layer), FHLOF (the lowest observed frequency of the high-angle ray along one-hop F

65 layer), FLLOF (the lowest observed frequency of the low-angle ray along one-hop F

66 layer), and 2FMOF (the maximum observed frequency of the two-hop F layer) can be

67 scaled from an oblique ionogram. Among these the FMUF, known as basic MUF or 
68 Junction Frequency (JF) of one-hop F2 trace, is the most important one, who decides

69 the highest frequency at which a radio wave can propagate between given terminals by

70 ionospheric propagation alone (Pezzopane and Pietrella 2008). In an oblique ionogram,

71 the basic MUF corresponds with the frequency at which the high- and low-angle rays

72 join.

73 Due to the Earth's magnetic field, the ionosphere is a doubly refracting medium. It

74 could be observed that two distinct ionospheric echo traces exist in an oblique ionogram,

75 which are so-called ordinary $(\mathrm{O})$ and extraordinary $(\mathrm{X})$ wave traces. The JFs of the O-

76 and X-waves are obviously different. Although it is sufficient to only extract O-wave

77 JFs for most applications, there are still some occasions that the O- and X-wave

78 parameters need to be accurately extracted simultaneously, such as studying the

79 ionospheric response to the geomagnetic field, improving the accuracy of lower

80 ionization layer electron density of ionospheric inversion (Storey 1960), etc. However,

81 due to the influences of random time-varying nature of the ionosphere and harsh

82 electromagnetic environment, the F-layer O- or X-wave trace is usually submerged by

83 noise or interference in oblique ionograms. Then it is difficult to obtain sufficient 
84 scalable ionograms to provide a physically relevant measurement of the O- and X-wave

85 JFs. In this case, it is useful to master the specific characteristics of the separation in JFs

86 between $\mathrm{O}$ - and $\mathrm{X}$-wave, which can be helpful in automatically acquainting $\mathrm{O}$ - and

87 X-wave parameters.

88 As early as 1970, Bradley, Eccles and King(1970) pointed out that the JF separation

89 between $\mathrm{O}$ - and $\mathrm{X}$-waves merited further investigation, and should be established how it

90 varied with path, latitude, mode order and direction of propagation. Since then, many

91 scholars have carried out in-depth research. Agy and Davies (1959) found that this

92 separation decreased with an increase in distance for the case of east-west path, which

93 gradually changed from $0.7 \mathrm{MHz}$ of vertical sounding scene to about $0.2 \mathrm{MHz}$ on a

$942400 \mathrm{~km}$ path. Magneto-ionic splitting of O- and X-components was also studied by

95 Kopka and Moller (1968) using ray tracing and a flat Chapman layer. Results were

96 given for a $2000 \mathrm{~km}$ path as a function of both magnetic latitude and magnetic azimuth,

97 showing that: (1)the magneto-ionic splitting was less for an east-west path than a

98 north-south path; (2)For a transmission in the direction of the magnetic meridian

99 (north-south path), the magneto-ionic splitting decreased with increasing magnetic 
100 latitude;(3)For a transmission transverse to the meridian, the splitting was zero at the

101 equator and increased towards the pole. Davies (1990) presented the relationship

102 between the JFs of the O- and X-wave (referred as $f_{O}$ and $f_{X}$ separately) reflected for

103 the cases of trans-equatorial and magnetic east-west propagation, which were $f_{X}-$

$104 f_{O} \approx f_{H}^{2} / 2 f_{O}$ and $f_{X}-f_{O} \approx f_{H} \cos I$ ( $I$ is the magnetic dip, $f_{H}$ is the electron

105 gyrofrequency) respectively. An explicit formula about the separation in JFs between

106 O- and X-wave (abbreviated as $f_{X}-f_{O}$ ) as a function of local magnetic dip and

107 azimuth of propagation was obtained by Bennett and Dyson (1994) for long paths, and

108 they also showed how to use analytic ray tracing to determine the separation for shorter

109 paths. Lundborg et al. (1995) found that the separation varied with propagation distance

110 and maximum observed frequency (MOF), changing between $0.7 \mathrm{MHz}$ when MOF was

111 low (about 5MHz) and $0.4 \mathrm{MHz}$ when MOF was high (about $15 \mathrm{MHz}$ ) for propagation

112 over the path Kiruna to Uppsala.

113 So far, most scholars have analyzed the variability of the separation in JFs between O-

114 and $\mathrm{X}$-wave from different aspects, and there is still a lack of systematic and

115 comprehensive analysis. As a continuation of the study, the present paper focuses on the 
variation characteristics of the separation by investigating the dependences on local time,

117 season, geomagnetic activity, solar activity, direction and length of propagation,

118 O-wave JF and group path. The contributions of this article lie as follows.

119 (1) The dependences of the separation on local time, season, geomagnetic activity,

120 O-wave JF and group path are statistically analyzed based on observed oblique

121 ionograms over three mid-latitude paths within China. A general diurnal tendency

122 and a summer anomaly on east-west propagation are first proposed and discussed.

123 (2) The influences of the length of the propagation circuit, the direction of propagation

124 and the solar activity variability have been analyzed by a ray-tracing program. A

125 different variation pattern of the separation with the propagation length is presented

126 which is partly inconsistent with previous results.

127 This article is organized as follows. The source of the data and the plan of the

128 experiment are briefly described in section 2 . Then, the method of analysis and

129 statistical results obtained for two years period over three mid-latitude paths within

130 China are presented in section 3. In section 4, we discuss the presented results and

131 investigate effects of solar activity, direction and length of propagation on the 
separation. Finally, the conclusion is drawn in section 5.

\section{Data sets}

134 The data sets are collected by an oblique sweep-frequency pulse transmission

135 experiment carried out by the China Research Institute of Radio-wave Propagation

136 (CRIRP). In this experiment, three transmitters and one receiver were deployed to

137 establish three radio paths in the middle of China as shown in Figure 1. The three paths

138 were arranged with different orientations and basically the same length, about $1000 \mathrm{~km}$.

139 The receiver system located at Xi' an and three transmitters located at Lianyungang,

140 Deqing and Linchuan respectively. The configuration was beneficial in observing the

141 influence of the propagation directions on O- and X-wave JFs. Details of the radio links

142 are presented in Table 1. The geomagnetic azimuths of the paths range from $90.1^{\circ}$ to

$143141.6^{\circ}$, and correspondingly the propagation turns east-west propagation to

144 quasi south-north propagation.

145 The instruments used for this experiment which were designed and manufactured by

146 CRIRP were essentially the same as that used for sweep-frequency echo sounding of

147 vertical incidence covering the frequency range from 3 to $30 \mathrm{MHz}$ with a sweep rate of 
$14850 \mathrm{kHzs}^{-1}$. The main difference was that the antennas used to transmit and receive

149 signals were horizontally polarized log-periodic antenna and horizontal dipole antenna

150 in this case, while delta antenna and orthogonal inverted $\mathrm{V}$ antenna were used for

151 vertical sounding. Furthermore, the high precision Global Positioning System (GPS)

152 was employed to provide the system timing ensuring that transmitter and receiver

153 synchronized with an accuracy better than 10us and also allow absolute time of flight

154 measurements to be made.

155 The system used low power, pulse compression waveform and made extensive use of

156 digital signal processing techniques in order to measure channel parameters and obtains

157 the oblique ionograms. The maximum output power of the transmitter was 400W. A

158 maximal length pseudo noise sequence (M-sequence) of length 255 or 511 chips

159 modulated on to a bi-phase PSK carrier was employed to offer a processing gain of

160 about $24 \mathrm{~dB}$ or $27 \mathrm{~dB}$. Each code sequence lasted $6.375 \mathrm{~ms}$ or $12.775 \mathrm{~ms}$ giving a signal

161 bandwidth of $40 \mathrm{kHz}$ and the range resolution corresponding to $7.5 \mathrm{~km}$. The repetition

162 period of the pulse was $25 \mathrm{~ms}$. In order to improve the signal-to-noise ratio, the coded

163 pulses were sent 5 times at each frequency. 
164 The experiment was organized and carried out during two periods, 2008 interval and

1652020 interval, covered the minimum of solar cycle 24 and 25 respectively. The first

166 period ran during January-December in 2008, and the measurements were performed

167 round the clock during local time 8:00-19:00 every day with a 15 minutes interval,

168 which means the system operated only on daytime and one oblique ionogram for each

169 path was generated every 15 minutes. On the second period, measurements were

170 performed once a quarter for $24 \mathrm{~h}$ a day during 2020 . The junction frequencies of the O-

171 and $\mathrm{X}$-wave were manually extracted for every oblique ionogram over each path except

172 that the O- and X-mode traces cannot be successfully identified. A data base of 78783

173 recordings was built up during the campaign. In addition, the 3-hourly Kp index in the

174 corresponding time period was obtained to analyze the relationship between the

175 separation $f_{X}-f_{O} f_{X}-f_{O}$ with the geomagnetic activity, which was provided in the

176 Web site: http://wdc.kugi.kyoto-u.ac.jp/.

1773 Data analysis and results

\section{$178 \quad 3.1$ Direction Variability}

179 The probability density of the separations in JFs between O- and X-wave is firstly 

investigated to obtain an overall picture of Magneto-ionic splitting variation over three

181 radio links, as shown in Figure 2. For Link 1, a magnetic east-west propagation path,

182 the separation $f_{\mathrm{X}}-f_{\mathrm{O}}$ changes between $0.1 \mathrm{MHz}$ and $0.5 \mathrm{MHz}$, and mainly

183 concentrates in $0.15-0.35 \mathrm{MHz}$. The separation for Link 2 (the southeast-northwest

184 propagation path) varies from $0.2 \mathrm{MHz}$ to $0.7 \mathrm{MHz}$ with a mean value of $0.4 \mathrm{MHz}$ and for

185 Link 3(the quasi south-north propagation path) it varies between $0.3 \mathrm{MHz}$ and $1.0 \mathrm{MHz}$

186 with a mean value of $0.67 \mathrm{MHz}$. It is obvious that the $f_{\mathrm{X}}-f_{\mathrm{O}}$ increases as the

187 propagation direction deviating from the direction of east-west. This statistical feature is

188 consistent with previous research results that the magneto-ionic splitting is less for an

189 east-west path than a north-south path.

\section{$190 \quad 3.2$ Local-Time Variability}

191 The JFs data observed in the two periods are grouped together and are binned as a

192 function of local time (LT) with the interval of $1 \mathrm{~h}$. The variation of the separation $f_{\mathrm{X}}-$

$193 f_{\mathrm{O}}$ over three radio links against LT is presented separately in Figure 3. It can be found

194 that the diurnal tendencies of the separation on the three paths exhibit different patterns.

195 The separations on Link 1 and 2 rise after sunrise, have peak near local noon, fall during 
196 the afternoon, and then rise again reaching a high level by local midnight or early

197 morning with a maximum at nighttime. However, the separation on Link 3 presents no

198 obvious day-night asymmetry and varies around the mean value throughout the whole

199 day. It can be conclude that the magneto-ionic splitting on east-west propagation link is

200 more susceptible to LT variability comparing with north-south propagation. It is

201 necessary to stress that the variation in hourly average on Link 3 is less than (or

202 comparable with) the system frequency resolution.

\section{$203 \quad 3.3$ Seasonal Variability}

204 To evaluate the dependence of the separation $f_{\mathrm{X}}-f_{\mathrm{O}}$ on seasons, the JFs data are

205 sorted into the following four seasonal bins just as Wang et al.: summer (May, June,

206 July and August), spring (March and April), autumn (September and October), and

207 winter (January, February, November and October) (Wang et al. 2018). The hourly

208 mean of the separations in each bin is calculated for each radio link. The statistical

209 results are shown in Figure 4.The following common features could be found: (1) the

210 seasonal distribution pattern on Link 3 is obviously different from Link1 and 2;(2) the

211 daytime separations in summer are larger than other seasons on Link1 and 2, which has 
212 a deviation greater than $0.1 \mathrm{MHz}$, while there is no significant seasonal difference on

213 Link 3 where the hourly value mainly changes within $0.05 \mathrm{MHz}$; (3) For Link1 and 2,

214 the largest separation of the day occurs at night in most seasons except summer, when

215 the most dominant separations present during daytime(denoted as summer anomaly).

216 This statistical feature suggests that the seasonal variability of the separation on

217 east-west propagation link is more prominent than that on north-south path.

\section{$218 \quad 3.4$ Geomagnetic Activity}

219 Diurnal variation of 3-hourly Kp index is employed for studying the dependence of the

220 separation $f_{\mathrm{X}}-f_{\mathrm{O}}$ on geomagnetic variability. The data are categorized into two sets

221 of quiet and disturbed conditions of geomagnetic activity. If the maximum value of the

222 3-hourly Kp index for a day is greater than 4, the day is considered as a disturbed day

223 and the data observed during the day is classified into the disturbed set. Figure 5 shows

224 the daily max Kp indices in 2008 and 2020. Observations from Figure 5 show that there

225 are only about ten percent of the time occurs geomagnetic disturbance during the

226 experimental period. The mean value of the separations in each bin is calculated

227 separately for each radio link showing in Figure 6. The separation in geomagnetic 
230 is no pronounced dependence on Link 3.

\section{$231 \quad 3.5$ O-wave JF and group path Variability}

232 In order to analyze the correlation between the separation $f_{\mathrm{X}}-f_{\mathrm{O}}$ and the O-wave $\mathrm{JF}$

233 and group path, the JFs are binned as a function of $\mathrm{O}$-wave $\mathrm{JF}$ from $3 \mathrm{MHz}$ to $22 \mathrm{MHz}$ in

234 steps of $3 \mathrm{MHz}$ and as a function of O-wave group path from $1000 \mathrm{~km}$ to $1450 \mathrm{~km}$ in steps of $50 \mathrm{~km}$. The statistical mean of the separations is calculated as the ratio of the summation of the observed separations to the total number of separations in each bin.

237 Figures 7 and 8 show the O-wave JF and group path variation compared with the

238 separation on three radio links. Observations from Figure 7 show that for all cases the

239 separation initially decreases with increasing O-wave JF, but for the cases of Link 2 and

2403 , the separation decreases to a minimum and then increases very slowly with a total

241 deviation less than the frequency resolution. Figure 8 shows that the separation

242 increases with a decrease in O-wave group path on Link1 and 2, and slightly decreases

243 on Link3. It is evident that the separation in the case of east-west path is more 
244 susceptible to the O-wave JF and group path.

\section{Discussions}

246 In the above analysis, we have focused on the statistical characteristics of the separation

247 in JFs between O- and X- wave in the mid-latitude China region. The statistical mean,

248 local time, season, geomagnetic activity, O-wave JF and group path dependences are

249 analyzed. The presented results are, in general, consistent with the previous theoretical

250 and experimental results (Agy and Davies 1959; Kopka and Mo 1ler 1968; Davies 1990;

251 Bennett et al. 1994; Lundborg et al. 1995). However, some new findings in the above

252 statistical investigation are worth further discussions.

\section{$253 \quad 4.1$ Case of east-west propagation}

254 The statistical investigations among the three paths show that the separation on

255 east-west path is really small, but its relative change is much more prominent and more

256 susceptible on local-time and seasonal variability. Figure 3 and 4 demonstrate that the

257 separation on east-to-west path regularly changes with local time. In general, the separation gradually increases after sunrise with one maximum near local noon, then 
259 slowly decreases before sunset, and grows up until reaching another maximum at

260 midnight or presunrise, and then decreases again with one minimum between local time

261 7:00 and 8:00. Among these, the most striking feature is summer anomaly showing that

262 the separation during daytime is more prominent than that at night in summer, while it

263 is the opposite in other seasons. Based on the relationship between the frequencies of

264 the $\mathrm{O}$ - and $\mathrm{X}$-waves reflected at the same equivalent height for the cases of magnetic

265 east-west propagation, which is $f_{X}-f_{O} \approx f_{H}^{2} / 2 f_{O}$, this phenomenon cannot be well

266 explained. Since the relationship implies that the frequency separation decreases with

267 increasing wave frequency, while the highest daily O-wave JF in summer occurs during

268 the daytime like other seasons, as shown in Figure 9. Therefore, the seasonal

269 dependence of the separation shows a more complex situation.

270 The typical oblique ionograms in summer daytime are reviewed to seek for possible

271 explanations, as shown in Figure.10. Observations reveal that when the low-angle trace

272 of the F-layer is upturned and the available frequency band becomes narrower, the

273 separation gets larger. One possible interpretation is that the existence of the completely

274 developed F1-layer or strong Es-layer increases the transmission length of F2 mode 
275 which further increases the difference in raypath between $\mathrm{O}$ - and $\mathrm{X}$-wave, thereby the

276 frequency difference between O- and X-wave increases. In order to verify this idea, a

277 ray tracing program is adopted to simulate the changes in ray path when the F1-layer

278 exists or not. The ionospheric model used in this verification is 'IRI-2016' with the F1

279 model being set to 'Scotto-1997 no L' or 'None'. Figure 11 presents the synthesized

280 oblique ionograms with and without F1-layer. It can be seen that when there is a

281 F1-layer the separation gets larger of $0.025 \mathrm{MHz}$ compared with the condition having no

282 F1-layer.

283 Further statistics on the hourly mean values of the O-wave JF and group path in each

284 season were described in Figure 9. It is obvious that the lowest O-wave JF during

285 daytime occurs in summer, and the largest O-wave group path also presents during the

286 summer day. Combined with the previous points on the relationships between the

287 separation and O-wave JF and group path, the seasonal distribution pattern of the

288 separation can be reasonable understood.

289 Another noteworthy phenomenon is that the separation on east-west path is slightly

290 weakened under active geomagnetic conditions compared to quiet conditions. 


\subsection{Case of quasi north -south propagation}

292 Our statistics confirms that the separation on quasi north-south propagation path is the

293 most significant among the three links, which agrees with previous studies. This means

294 that the magneto-ionic splitting on north-south path is more prominent than that on

295 east-west path. Furthermore, the separation does not vary significantly with the local

296 time and season, and also have weak correlation with O-wave JF and group path. It may

297 be explained by Davies's relationship between the frequencies of the O- and X-wave for

298 the cases of quasi-longitudinal propagation, that is $f_{X}-f_{O} \approx f_{H} \cos I$. The

299 quasi-longitudinal approximation is fairly good near the maximum frequency on

300 north-south path (Davies 1990). The relationship shows that the separation is mainly

301 determined by the gyrofrequency and the magnetic dip, and is hardly affected by the

302 ionospheric activity. However, there is no obvious abnormality in the mean value of the

303 separations during the period of geomagnetic disturbance. An in-depth study is needed

304 to interpret this phenomenon, since the occurrence of magnetic storms will cause a

305 strong global simultaneous magnetic disturbance which may cause fluctuations in

306 geomagnetic intensity. 


\section{3 solar activity variations}

308 Aforementioned analysis shows that the separation on east-west path is susceptible on

309 ionospheric variability, and it cannot be interpreted well by Davies's relationship.

310 Davies (1990) pointed out that the left-handed O-wave and the right-handed X-wave

311 introduced by magneto-ionic splitting traveled different paths in the ionosphere and it

312 was not possible to integrate analytically to obtain the precise ray path, but it was

313 practical to do so by means of point-to-point ray tracing.

314 Based on the use of an equivalent operating frequency, Bennett (Bennett et al. 1994)

315 provided an analytic ray tracing method to determine the separation. The simple form of

316 the separation is given as (Dyson and Bennett 1979, 1980 )

$$
f_{x}-f_{o}=\frac{1}{2} f_{H}\left[h_{x}(\Theta, W)-h_{o}(\Theta, W)\right]
$$

318 Where $\Theta$ is the angle between the wave normal direction and the direction of the

319 magnetic field, $W=(X-1) / Y, \quad \mathrm{X}=\frac{f_{N}^{2}}{f^{2}}, Y=\frac{f_{H}}{f}$, and

320

$$
h_{o}(\Theta, W)=\frac{C^{2}\left\{\left(1+\frac{4 C^{2} W^{2}}{S^{4}}\right)^{1 / 2}-1\right\}}{-\frac{1}{2} \frac{1}{W}\left\{1+C^{2}+S^{2}\left(1+\frac{4 C^{2} W^{2}}{S^{4}}\right)^{1 / 2}\right\}-\frac{2 C^{2} W}{S^{2}}}
$$




$$
h_{x}(\Theta, W)=-\frac{C^{2}\left\{\left(1+\frac{4 C^{2} W^{2}}{S^{4}}\right)^{1 / 2}+1\right\}}{-\frac{1}{2} \frac{1}{W}\left\{1+C^{2}-S^{2}\left(1+\frac{4 C^{2} W^{2}}{S^{4}}\right)^{1 / 2}\right\}-\frac{2 C^{2} W}{S^{2}}}
$$

322 where $C=\cos \Theta, S=\sin \Theta$.

323 A 3-D magnetoionic Hamiltonian ray tracing toolbox(PHaRLAP) has been developed

324 by the Defence Science and Technology Organization(DSTO) which can be used to

325 calculate the JFs of the $\mathrm{O}$ and $\mathrm{X}$ polarization modes(Cervera and Harris 2014).

326 To analyze the influence of solar activity on the separation, the performance of the two

327 methods mentioned above is first demonstrated, assessing its consistency with

328 experimental data. Our simulation is based on the IRI-2016 and IGRF-12 models, and

329 the 12-month moving averages of sunspot numbers during the two experimental periods

330 are adopted. A typical comparison is shown in Figure. 12 with blue line represents

331 monthly mean diurnal variation of the measurements, red line represents results

332 calculated by 3-D ray tracing method, and magenta line represents results calculated by

333 Bennett's method. It is seen from this figure that the separation is little overestimated by

334 3-D ray tracing method(less than $0.05 \mathrm{MHz}$ ) and obviously underestimated by Bennett's

335 method during daytime, and the results calculated using 3-D ray tracing method show 
better agreement in diurnal variation tendency with measurements compared to the

337 results from Bennett's method. So, it is a wise choice to make use of 3-D ray tracing for

338 analyzing the dependence of the separation on solar activity.

339 The diurnal variations of the separation are conducted on Link 1 covering three levels of

340 solar activity $(\mathrm{R}=10,70$ and 120, respectively represent the low, medium and high solar

341 activity) and four months (January, April, July and October, characterize four different

342 seasons), as shown in Figure 13.

343 It is observed that the double-peak variation of the separations with one maximum in

344 local noon and another maximum in local midnight or presunrise is favored for all solar

345 activities in different seasons. In January and October, the separation at night is more

346 prominent than that during daytime, and the maximum at night occurs at low solar

347 activity while the largest value during daytime generally presents at high solar activity.

348 In April, the separation during low solar activity phase is higher at night and slightly

349 lower in daytime which is consistent with the observations over Link 1, while the

350 separations during medium and high solar activities are larger in daytime than that at

351 night. In July, the separation during the day is more obvious when compared with that 
352 at night for all solar activities. Our results suggest that the separation is mainly affected

353 by $\mathrm{O}$-wave JF at night and shows positive correlation with $\mathrm{O}$-wave group path during

354 daytime.

\section{4.4 Dependences on length and direction of propagation}

356 In order to investigate sensitivity to the length and direction of propagation, calculations

357 were performed for different path configurations with a central receiver at Xi'an. The

358 results obtained by 3-D ray tracing method discussed in this paper are shown in Figure

359 14. The figure above shows the variability of the separation as a function of the

360 direction of propagation for two different transmission lengths that are $1000 \mathrm{~km}$ and

$3612000 \mathrm{~km}$, while the figure below shows the variability of the separation against with the

362 length of propagation for four different transmission azimuth angles of $45^{\circ}, 90^{\circ}$,

$363145^{\circ}$ and $180^{\circ}$. As we can see, the separation varies approximately as the cosine function

364 of the propagation direction with maximums at north-south direction and minimums at

365 east-west direction in mid-latitude China region. Due to the different transmission

366 directions, the separations on radio links with ground range greater than $500 \mathrm{~km}$ present

367 obviously distinct patterns. For east-west propagation path, the separation decreases to a 
minimum near ground range of $2000 \mathrm{~km}$ and then increases very slowly with increasing

369 ground range, while for north-south propagation path, it gradually increases with

370 increasing ground range. The relationship of the separation with transmission length is

371 partly inconsistent with results simulated by Bennett et al. (1994) which show a

372 tendency to decrease below $1000 \mathrm{~km}$ and then increase very slowly for case with

373 azimuth equal to 0 (of north-south propagation). The possible reason for this difference

374 may be the inaccuracy of method and ionospheric model.

\section{$375 \quad 5$ Summary and conclusions}

376 We have presented a statistical and simulation study on the separation in JFs between O

377 and $\mathrm{X}$ wave based on oblique sounding data recorded by three mid-latitude paths within

378 China and 3-D ray tracing program. The dependences on local time, season,

379 geomagnetic activity, solar activity, direction and length of propagation, $\mathrm{O}$ wave JF and

380 group path are observed. The major conclusions are summarized as follows:

381 (1) The separation on east-west path is susceptible on ionospheric variability, which is

382 inconsistent with previous analysis that the separation does not depend strongly on

383 the ionospheric plasma distribution (Bennett et al. 1994), while the separation on 
north-south path does not show significant correlation with local time and season variations.

(2) The separation on east-west path is strongly dependent on the local time, season and solar activity. There is a general diurnal tendency, to rise after sunrise, have a peak near local noon, fall during the afternoon, and then rise again reaching a high level by local midnight or early morning. The most striking feature is summer anomaly showing that the separation during daytime is more prominent than that at night in summer, while it is the opposite in other seasons. One possible interpretation is the effect of refraction in completely developed F1-layer or strong Es-layer.

(3) The separation on east-west path is slightly weakened under active geomagnetic conditions compared to quiet conditions. However, there is no pronounced dependence on north-south path.

(4) The separation varies approximately as the cosine function of the propagation direction owning two maximums at north-south direction and two minimums at east-west direction in mid-latitude China region. This feature is consistent with previous research results that the magneto-ionic splitting is less for an east-west path 

than a north-south path.

401 (5) The separations on different transmission azimuths present obviously distinct patterns. For east-west propagation path, the separation decreases to a minimum near ground range of $2000 \mathrm{~km}$ and then increases very slowly with increasing ground range, while for north-south propagation path, it gradually increases.

405 Taking account care required in automatically extracting F2-layer O- and X-wave

406 parameters from oblique ionograms to achieve the best performance, a study for the 407 behavior of separation in JFs between O- and X-waves is always useful and worthy. Our 408 contribution is achieved through two aspects: Regarding experimental observation, the 409 general diurnal tendency and summer anomaly of the separation are firstly proposed;

410 regarding simulation, provides interesting insights into the dependences of the

411 separation on solar activity, length and direction of propagation and gains a different 412 conclusion from previous analysis.

413 The future work will be to improve frequency resolution and investigate the correlation 414 between the separation and geomagnetic activity. 


\section{Declarations}

416 Ethics approval and consent to participate

417 Not applicable.

418 Consent for publication

419 Written informed consent was obtained from study participants for

420 participation in the study and for the publication of this report and any

421 accompanying images. Consent and approval for publication was also

422 obtained from Wuhan University and China Research Institute of

423 Radio-wave Propagation.

424 List of abbreviations

$425 \mathrm{JF}$ : the frequency at which the high- and low-angle rays join;

426 O-mode or O-wave: the ordinary mode or wave;

427 X-mode or X-wave: the extraordinary mode or wave;

428 HF: high frequency;

429 LOF: the Lowest observed frequency; 
430 MOF: the Maximum observed frequency;

431 FMUF: the highest usable frequency of one-hop F layer;

432 FHLOF : the lowest observed frequency of the high-angle ray along one-hop

$433 \quad$ F layer;

434 FLLOF: the lowest observed frequency of the low-angle ray along one-hop F

435 layer;

436 2FMOF: the maximum observed frequency of the two-hop F layer;

437 CRIRP: China Research Institute of Radio-wave Propagation;

438 GPS: Global Positioning System;

439 DSTO: Defence Science and Technology Organization.

\section{Availability of data and materials}

441 The 3-hourly Kp index is available in the website: http://wdc.hugi.kyoto-u.ac.jp/.

442 Regretfully, the oblique ionograms data used in this manuscript cannot be shared

443 because they belonged to the China Research Institute of Radio-wave Propagation 
444 (CRIRP).

445 Competing interests

446 The author declares no competing interests

447 Funding

448 This work was supported by the National Natural Science Foundation of China (Grant

449 No.61931015), Innovation Group Project of Natural Science Foundation of Hubei

450 Province (Grant No.2021 CFA002) and Stable-Support Scientific Project of China

451 Research Institute of Radiowave Propagation(Grant NO.A132011W16). The funds

452 from Grant No. A132011W16 were used for data collection and analysis. The funds

453 from Grant Nos. 61931015, 2021 CFA002 were used for manuscript preparation.

\section{Authors' contributions}

455 SFJ designed the study, analyzed the data and wrote the manuscript. WXR, ZHB and

456 ZB contributed related analysis on data. BPP contributed related analysis on simulation.

457 ZB and CHY helped with the text of the paper, particularly with the introduction and

458 comparison with previous works. All coauthors contributed to the revision of the draft 
manuscript and improvement of the discussion. All authors read and approved the final

460 manuscript.

\section{Acknowledgements}

462 The authors acknowledge the Data Center of the China Research Institute of Radiowave

463 Propagation for help with ionograms collection. The authors would like to thank Dr.

464 Shuji Sun and Dr. Boya Li for proofreading this manuscript. The authors would also

465 like to thank the anonymous referee for the useful comments and suggestions for

466 improving the paper.

\section{Authors' information}

468 FengJuan Sun, is currently a Ph.D. student at Wuhan University. She also is a Senior

469 Engineer at the China Research Institute of Radiowave Propagation. She has authored

470 and coauthored 6 patents and over 8 journal articles. Her current research interests are in

471 ionospheric radiowave propagation and its influence on HF radar. Dr. XianRong Wan is

472 currently a Professor and Ph.D. candidate supervisor with Wuhan University whose

473 main research interests include design of new radar system such as over-the-horizon 
474 radar, passive radar, and array signal processing. Dr. HongBo Zhang is currently a

475 senior engineer at the China Research Institute of Radiowave Propagation. He has

476 participated in the activities of the ITU-R study group 3 and has submitted about 3

477 contributions to the ITU-R SG3. Master Bao Zhou and HongYan Cao are currently

478 senior engineers at the China Research Institute of Radiowave Propagation. Their

479 research interests are in shortwave frequency forecasting, spectrum monitoring and

480 management. Dr. PanPan Ban is a Ph.D. student at Wuhan University. Her current

481 research interests are in ionospheric forcasting and ionospheric radiowave propagation.

482 Endnotes

483 Not applicable.

484 References

485 Agy V, Davies K (1959) Ionospheric investigations using the sweep-frequency pulse

486 technique at oblique incidence. Journal of research of the National Bureau of

487 Standards-D.Radio Propagation 63(2):151-174

488 Bennett JA, Chen J, Dyson PL (1994) Analytic calculation of the ordinary (O) and 
extraordinary $(\mathrm{X})$ mode nose frequencies on oblique ionograms. Journal of Atmospheric

490 and Terrestrial Physics 56(5):631-636

491 Bradley PA, Eccles D, King JW (1970) Ionospheric probing using pulsed radio waves

492 at oblique incidence. Journal of Atmospheric and Terrestrical Physics 32:499-516

493 Cervera MA, Harris TJ(2014) Modeling ionospheric disturbance features in

494 quasi-vertically incident ionograms using 3-D magnetoionic ray tracing and

495 atmospheric gravity waves. Journal of Geophysical Research:Space Physics

$496 \quad 119: 431-440$

497 Chemogor L F,Garmash K P,Guo Q,Luo Y,Rozumenko V T,Zheng Y(2020)

498 Ionospheric storm effects over the People's Republic of China on 14 May 2019: Results

499 from multipath muti-frequency oblique radio sounding. Advances in Space Research

500 66(2):226-242. https://doi.org/10.1016/i.asr.2020.03.037

501 Davies K (1990) Ionospheric radio. London, United Kingdom: The Institution of

$502 \quad$ Engineering and Technology

503 Dyson PL, Bennett JA(1979) General formulae for absorption of radio waves in the 
504 ionosphere. Journal of Atmospheric and terrestrial phsics (41):367-377

505 Dyson PL,Bennett JA(1980) A universal chart for use in relating ionospheric absorption

506 to phase path and group path. IEEE Transactions on Antennas and Propagation

$507 \quad 28(3): 380-384$

508 Heitmann AJ, Gardiner-Garden RS (2018) A Robust Feature Extraction and

509 Parameterized Fitting Algorithm for Bottom - Side Oblique and Vertical Incidence

510 Ionograms. Radio Science:115-134.doi:10.1029/2018RS006682

511 Ippolito A, Scotto C, Sabbagh D, Sgrigna V, Maher P (2015) A procedure for the

512 reliability improvement of the oblique ionograms automatic scaling algorithm.Radio

513 Science: 454-460. doi:10.1002/2015RS005919

514 Ippolito A, Scotto C, Francis M, Settimi A, Cesaroni C(2015) Automatic interpretation

515 of oblique ionograms. Advance in Space Research 55:1624-1629

516 Kopka H, Mo 1ler HGM (1968) MUF calcultions including the effect of the Earth's

517 magnetic field. Radio Science 1(3):53-56

518 Lundborg B, Bro ms M, Derblom H (1995) Oblique sounding of an auroral ionospheric 
519 HF channel. Journal of Atmospheric and Terrestrical Physics 1:51-63

520 Pezzopane M, Pietrella M (2008) Interobl:An interactive software tool for displaying

521 and scaling oblique ionograms. Computers \& Geosciences 34:1577-1583

522 Phanivong B, Chen J, Dyson PL, Bennet JA(1995) Inversion of oblique ionograms

523 including the Earth's magnetic field. J.Atmos.Terr.Phys 57:1715-1721

524 Rao NN (1973) A note on the analysis of oblique ionograms. Journal of Atmospheric

525 and Terrestrial Physics 35:1561-1563

526 Reily MH, Kolesar JD (1989) A method for real height analysis of oblique ionograms.

527 Radio Science 24:575-583. doi:10.1029/RS024i004p00575

528 Rogov DD,Vystavnoi VM, Blagoveshchenskaya NF, Baryshev PE, Kalishin ASK(2021)

529 Russian high-latitude network of oblique ionospheric sounding. Russian Meteorology

530 and Hydrology 46(4):217-224

531 Storey LRO (1960)The joint use of the ordinary and extraordinary virtual height curves

532 in determining ionospheric layer profiles. Journal of Research of the National Bureau of

533 Standards-D. Radio Propagation 64(2):111-124 
534 Wang N,Guo LX, Zhao ZW, Ding ZH, Lin LK(2018) Spread-F occurrences and

535 relationships with foF2 and h'F at low-and mid-latitudes in China. Earth Planets Space

536

537 Figure legends

538 Figure 1 Map showing positions of the radio paths used in the experiments; the

539 geomagnetic azimuth of the three paths are $90.1^{\circ}, 112.5^{\circ}$ and $141.6^{\circ}$ respectively

540 Figure 2 Probability density of the separations observed through three radio links

541 Figure 3 Local time variation of the separations observed through three radio links

542 Figure 4 Seasonal variation of the separations observed through three radio links; blue,

543 red, black and green lines represent spring, summer, autumn and winter, respectively

544 Figure 5 Daily max Kp indices in 2008 and 2020, red solid line indicates $\mathrm{Kp}=4$

545 Figure 6 Geomagnetic variability of the separations observed through three radio links

546 Figure 7 Correlation between the separation and the O-wave JF

547 Figure 8 Correlation between the separation and O-wave group path 
548 Figure 9 Variations in O-wave JF and group path with local time; blue, red, black and

549 green lines represent spring, summer, autumn and winter, respectively

550 Figure 10 An oblique ionogram at 12:50PM on August 12,2020

551 Figure 11 Synthesized oblique ionograms with and without F1-layer

552 Figure 12 Comparison of measured $f_{x}-f_{o}$ with calculated results obtained by 3-D ray

553 tracing and Bennett's method; blue line represents measurements, red line represents

554 results calculated by 3 -D ray tracing method, and magenta line represents results

555 calculated by Bennett's method

556 Figure 13 Solar activity variability of the separation simulated using 3-D ray tracing

557 method; blue line for low solar activity, red line for medium solar activity, and green

558 line for high solar activity

559 Figure 14 Relationship of the separation with length and direction of propagation; upper

560 diagram shows variability on direction of propagation, lower diagram shows variability

561 on length of propagation

562 Table legends 
563 Table 1 Detail of the radio links used in the investigation

564

565 
Figures

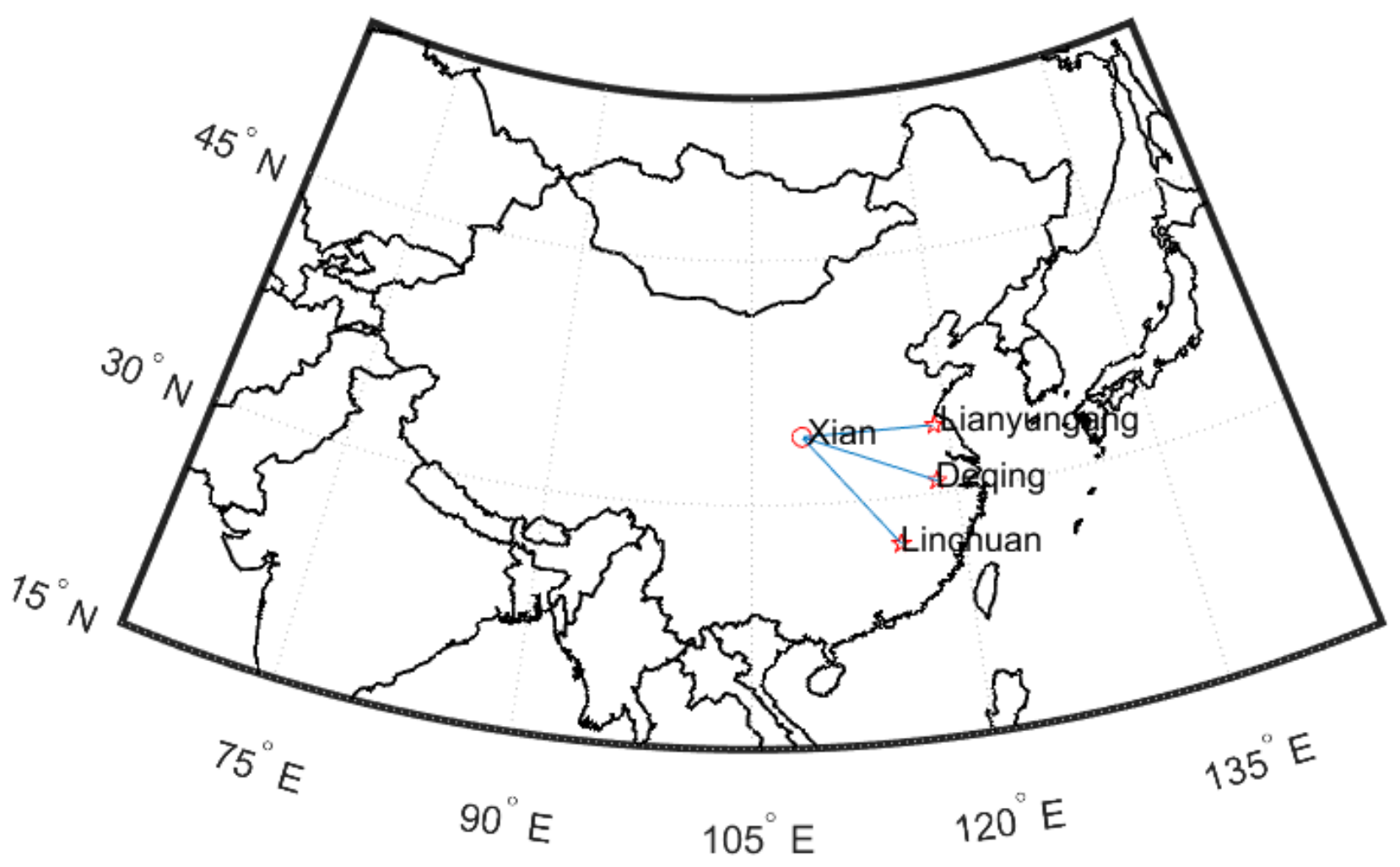

Figure 1

Map showing positions of the radio paths used in the experiments; the geomagnetic azimuth of the three paths are $90.1^{\circ}, 112.5^{\circ}$ and $141.6^{\circ}$ respectively 


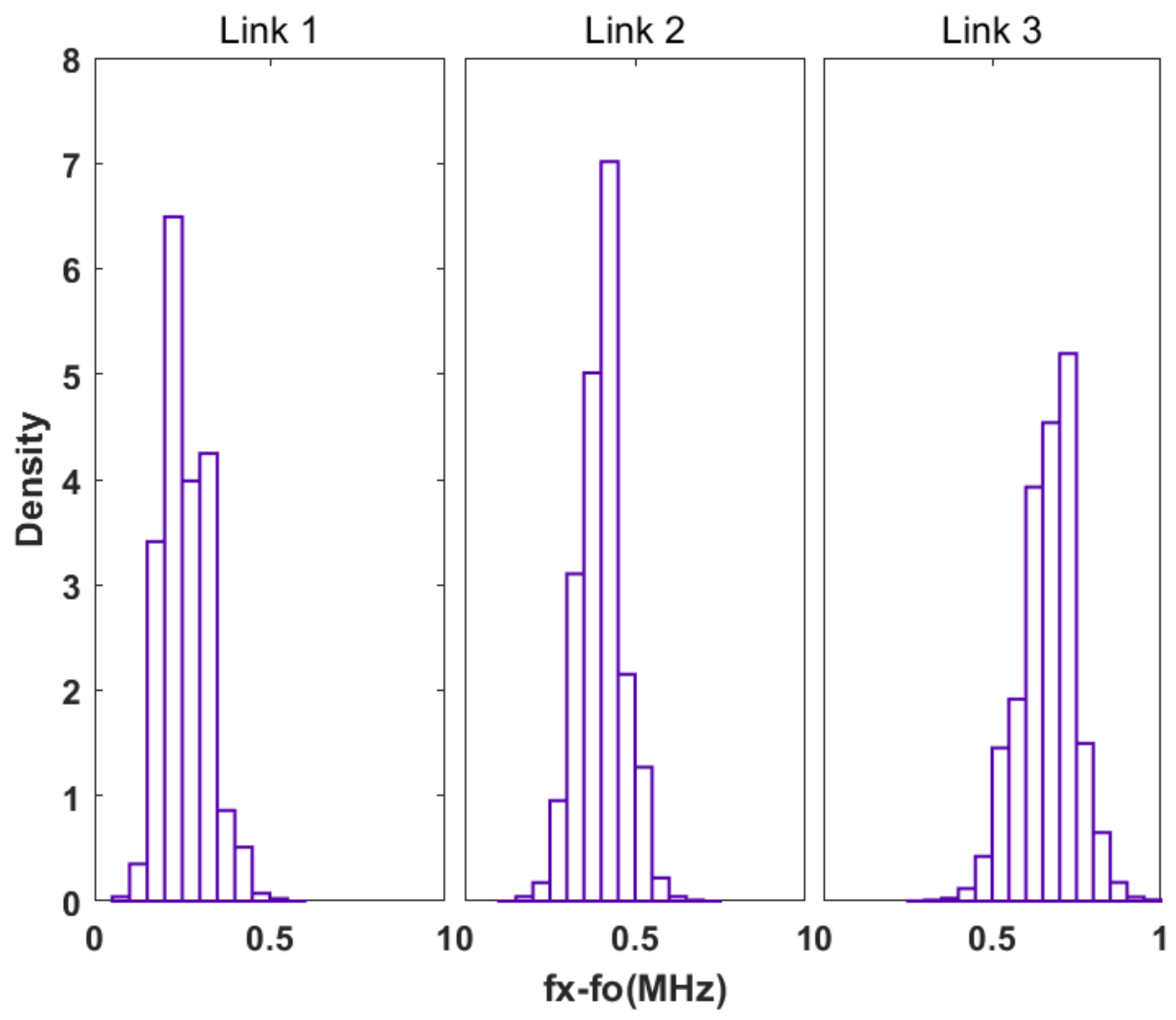

Figure 2

Probability density of the separations observed through three radio links

Figure 3

Local time variation of the separations observed through three radio links 


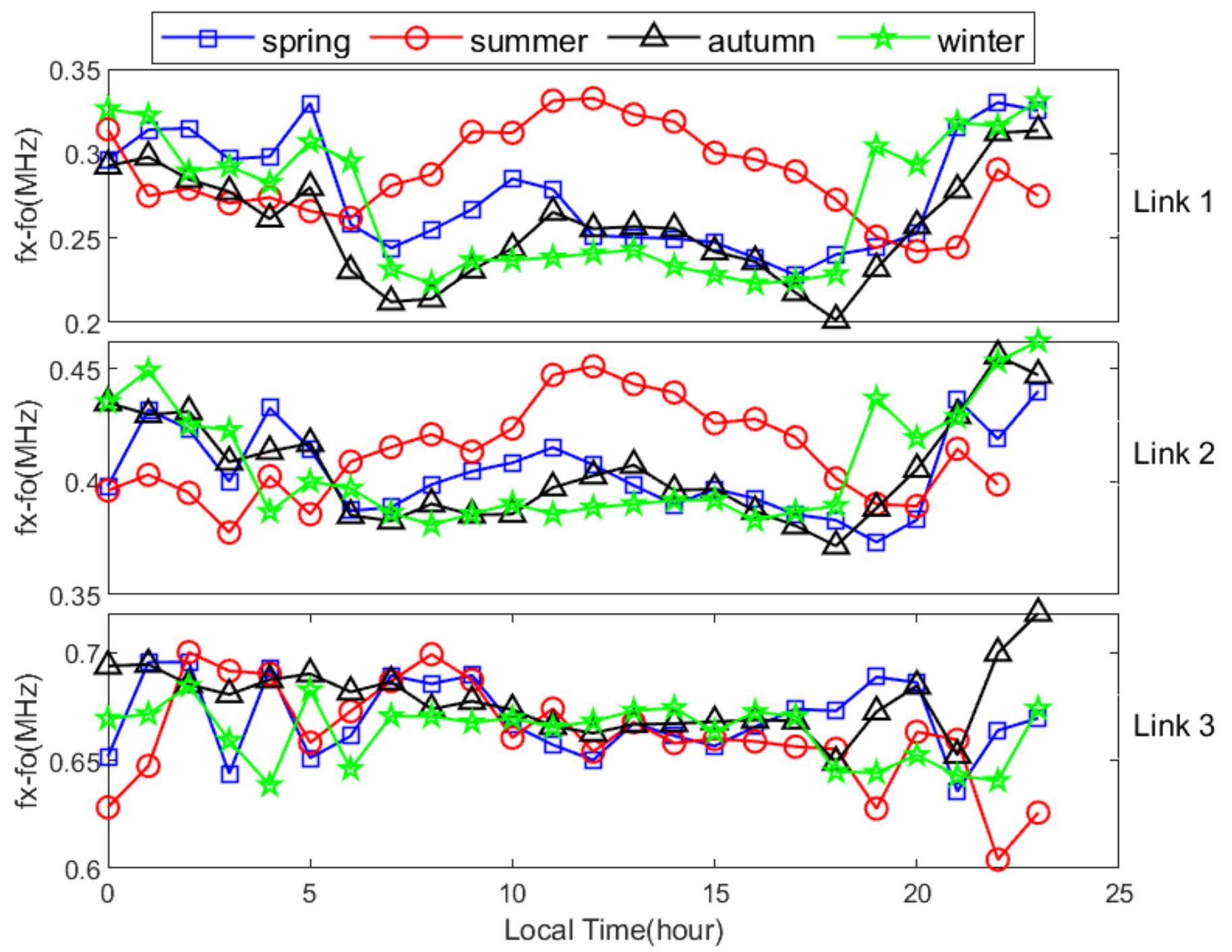

Figure 4

Seasonal variation of the separations observed through three radio links; blue, red, black and green lines represent spring, summer, autumn and winter, respectively 

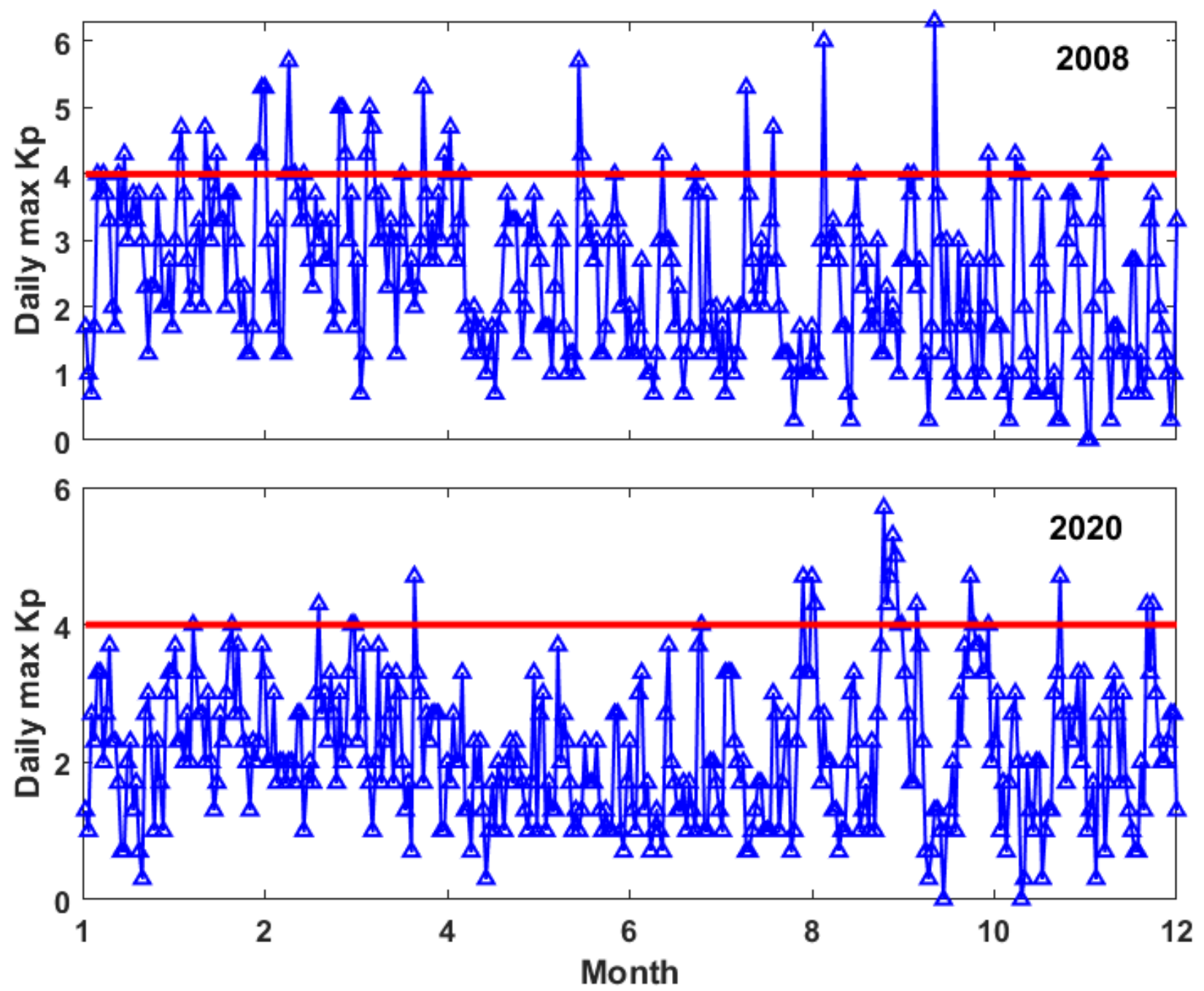

Figure 5

Daily max Kp indices in 2008 and 2020, red solid line indicates $\mathrm{Kp}=4$

Figure 6

Geomagnetic variability of the separations observed through three radio links 

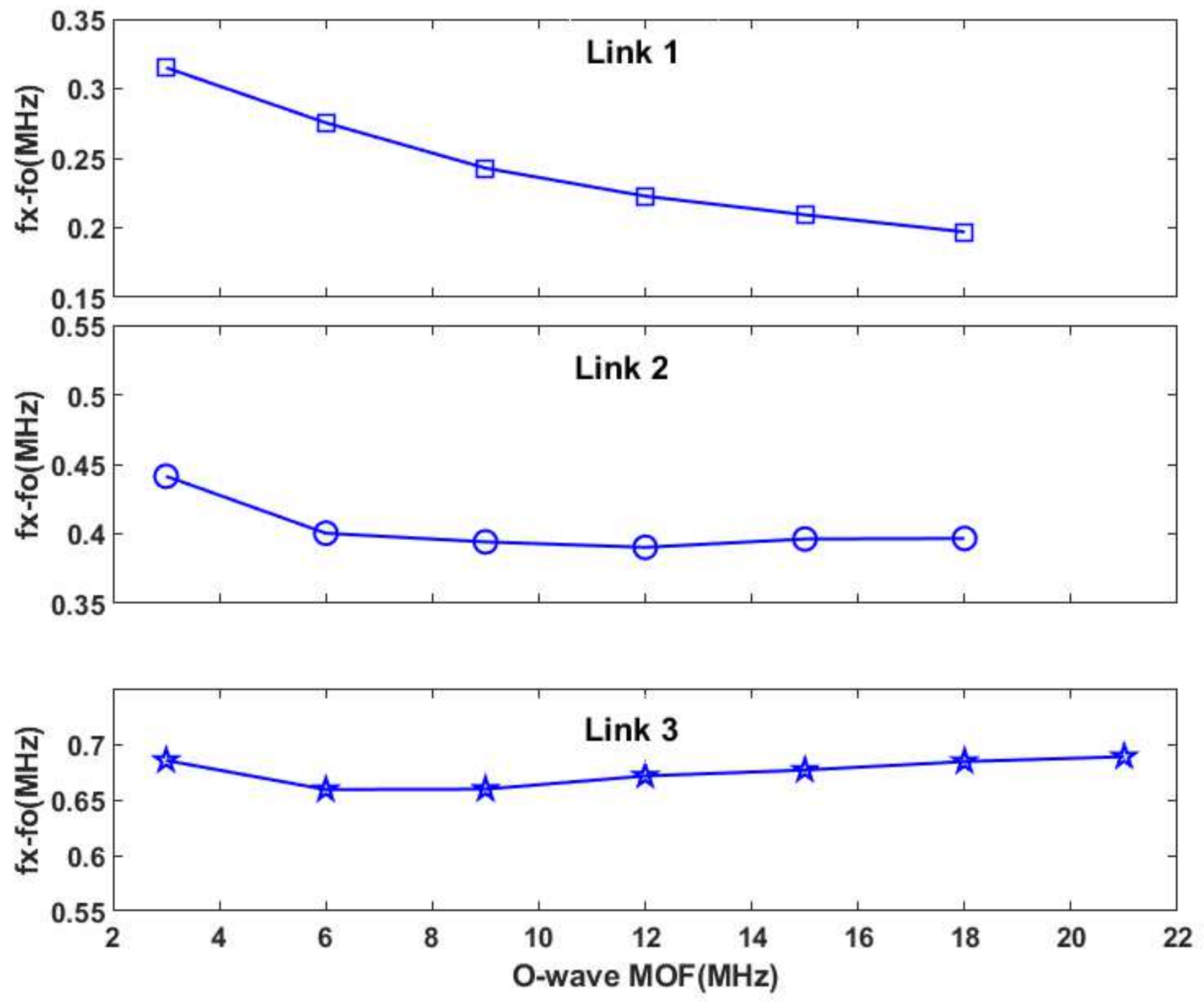

Figure 7

Correlation between the separation and the O-wave JF 


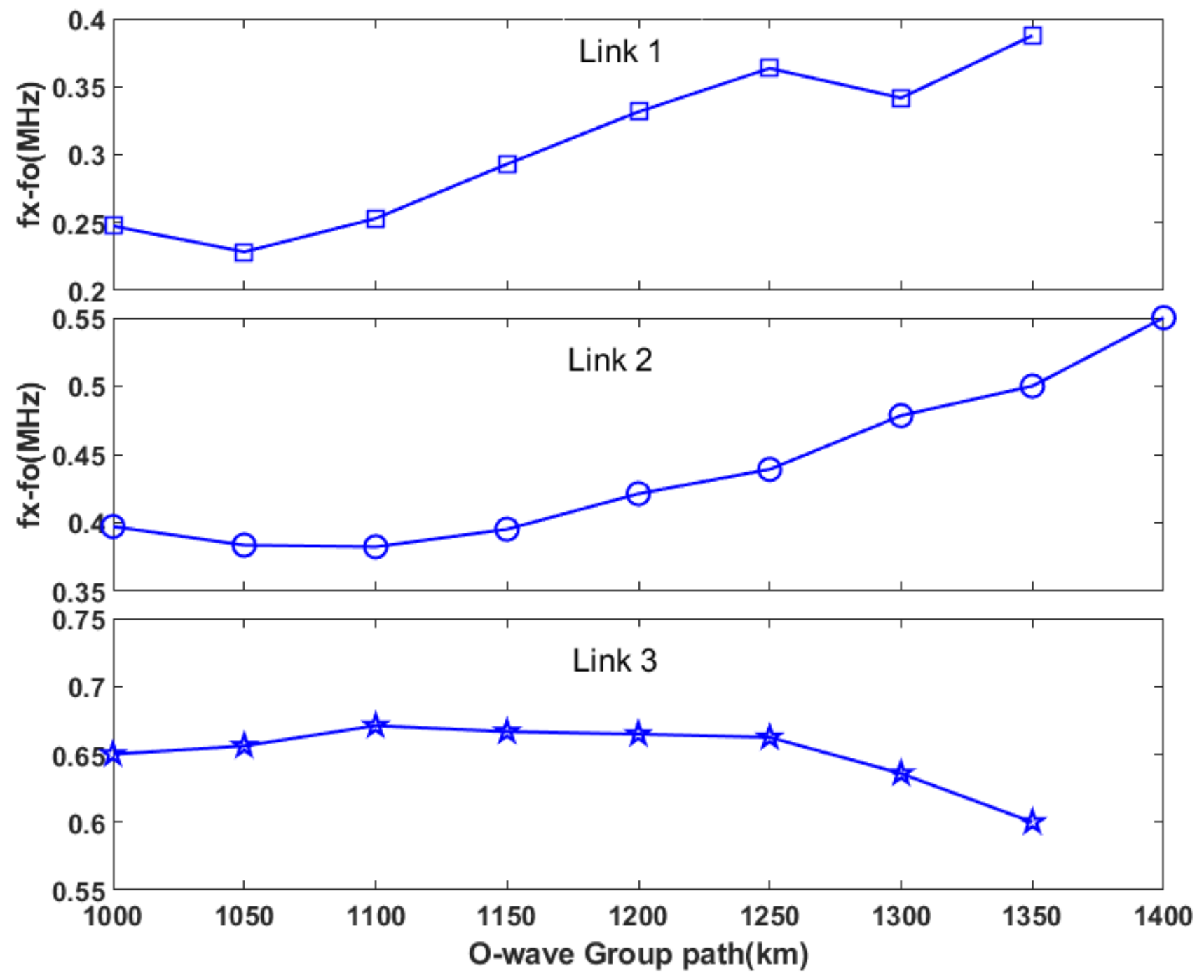

Figure 8

Correlation between the separation and 0-wave group path 

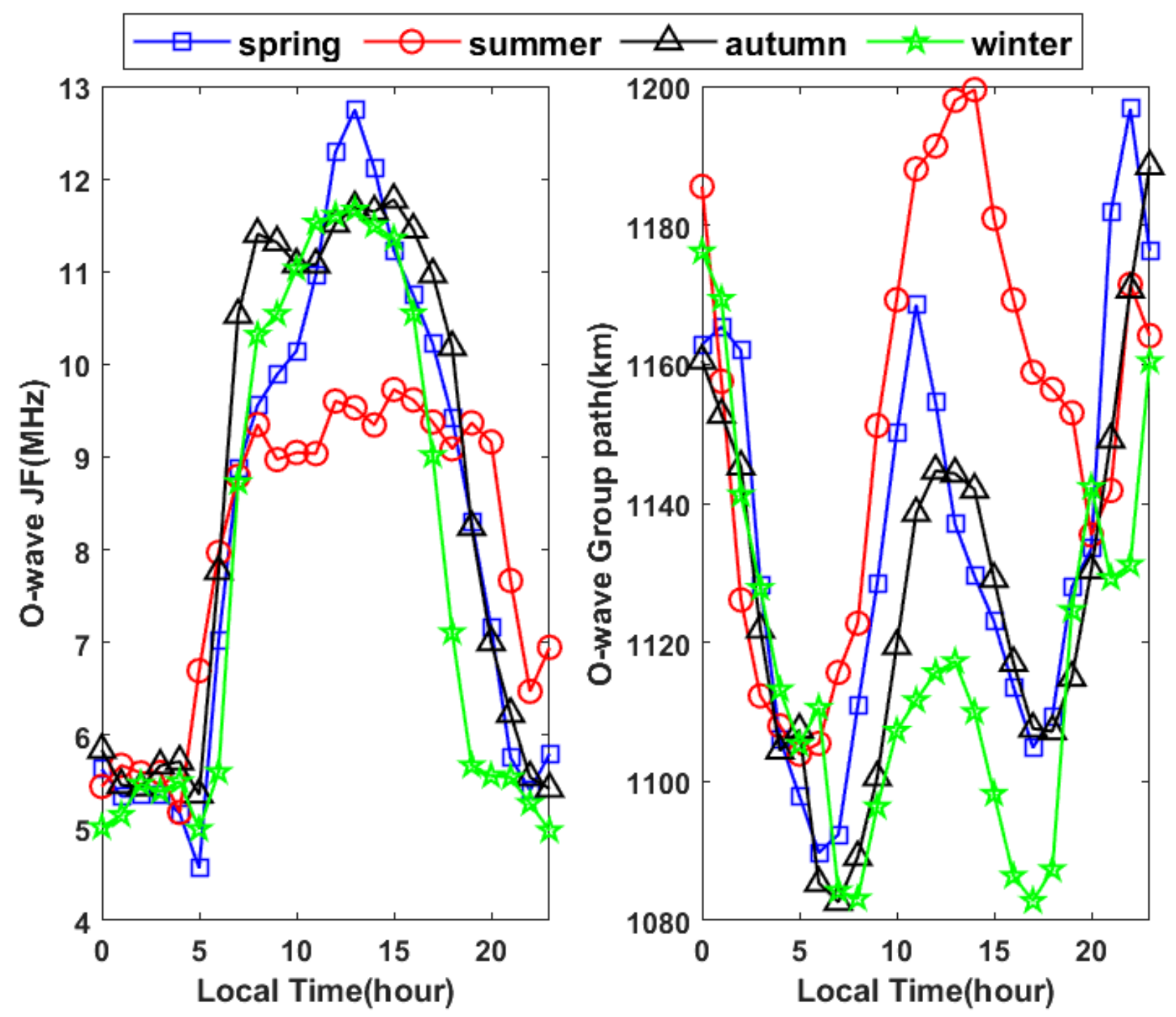

Figure 9

Variations in 0-wave JF and group path with local time; blue, red, black and green lines represent spring, summer, autumn and winter, respectively

Figure 10

An oblique ionogram at 12:50PM on August 12,2020

Figure 11

Synthesized oblique ionograms with and without F1-layer 
Figure 12

Comparison of measured $f_{x}-f_{o}$ with calculated results obtained by 3-D ray tracing and Bennett's method; blue line represents measurements, red line represents results calculated by 3-D ray tracing method, and magenta line represents results calculated by Bennett's method

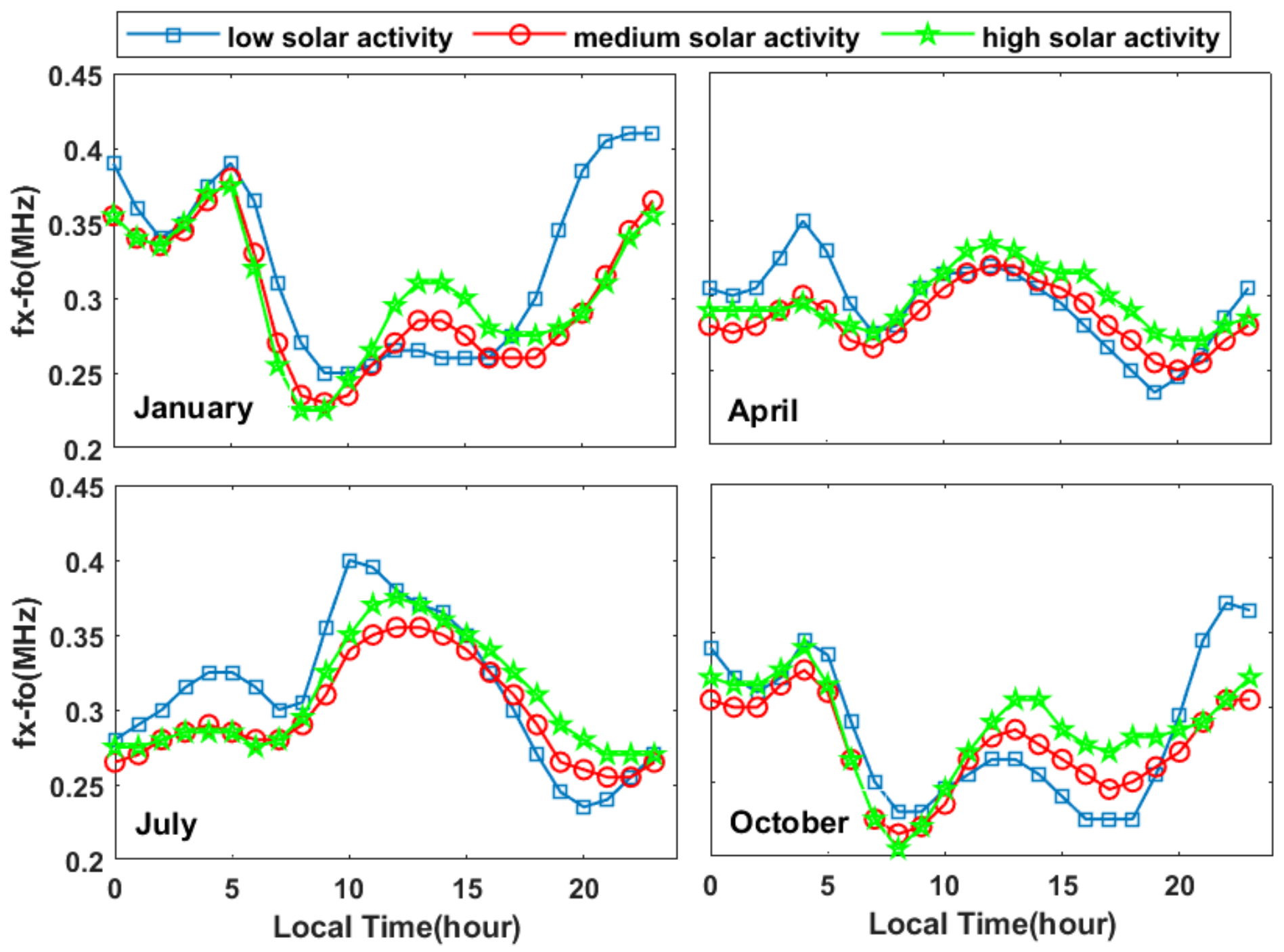

Figure 13

Solar activity variability of the separation simulated using 3-D ray tracing method; blue line for low solar activity, red line for medium solar activity, and green line for high solar activity 

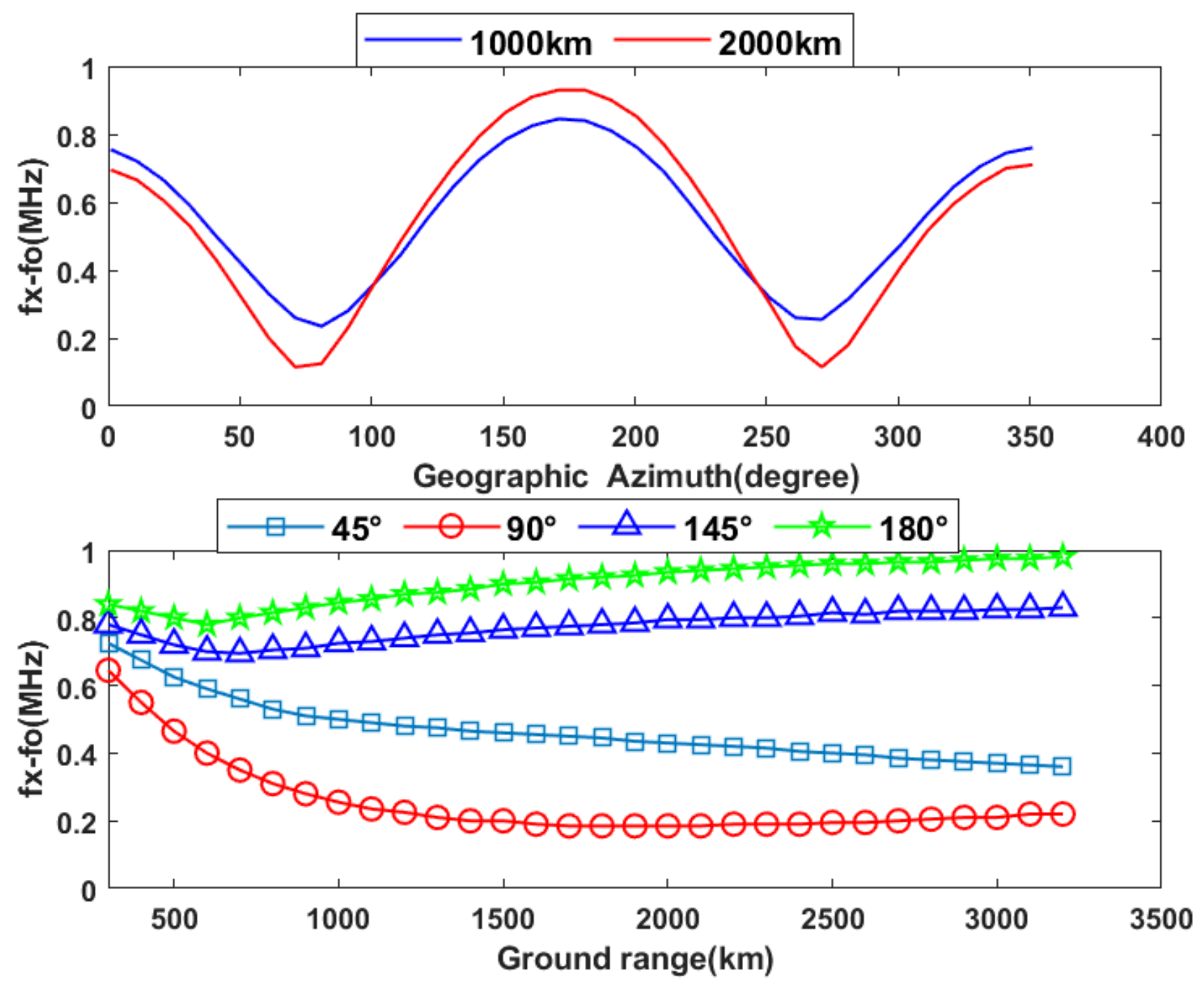

Figure 14

Relationship of the separation with length and direction of propagation; upper diagram shows variability on direction of propagation, lower diagram shows variability on length of propagation

\section{Supplementary Files}

This is a list of supplementary files associated with this preprint. Click to download.

- Graphicalabstractimage.jpg 